\title{
Strong-Field Molecular Ionization from Multiple Orbitals
}

\author{
Marija Kotur, ${ }^{1}$ Thomas C. Weinacht, ${ }^{1}$ Congyi Zhou, ${ }^{2}$ and Spiridoula Matsika ${ }^{2}$ \\ ${ }^{1}$ Department of Physics, Stony Brook University, Stony Brook, New York 11794, USA \\ ${ }^{2}$ Department of Chemistry, Temple University, Philadelphia, Pennsylvania 19122, USA
}

(Received 22 August 2011; published 28 November 2011)

\begin{abstract}
We demonstrate strong-field ionization from multiple orbitals of excited-state uracil molecules. The molecules are excited to the first bright state by an ultrafast laser pulse in the deep ultraviolet and then ionized with a strong-field laser pulse in the near infrared during ultrafast relaxation back down to the ground state. We measure time- and angle-dependent ion yields for multiple fragments created by strongfield ionization, and interpret the temporally and angularly resolved yields via $a b$ initio electronic structure calculations. We find that the angular distribution for the electron removed from the lowest unoccupied molecular orbital follows the symmetry of the molecular orbital, whereas ionization of the molecule by removing electrons from deeper bound orbitals is more complicated.
\end{abstract}

DOI: 10.1103/PhysRevX.1.021010

\section{INTRODUCTION}

Strong-field molecular ionization has drawn significant attention over the past few years as a probe of electronic orbitals and dynamics. It has been used as a critical component of imaging molecular orbitals [1-3] and to launch nuclear and electronic wave packets, which evolve on attosecond time scales [2,4-6]. There has been a focus on diatomic molecules because of their relative simplicity and the ability to align them relative to the laser polarization [7]. For the case of single-photon ionization, or recombination leading to harmonic emission, it was shown that the measurements reflect the Dyson orbital associated with the ionization [8]. In the case of tunnel ionization of groundstate diatomic molecules, while there is no direct formalism that relates the angle-resolved ionization yields to the molecular orbital structure, the ionization yield as a function of the angle between the laser polarization vector and the molecular axis has been shown to reflect the shape of the orbital from which an electron was removed, i.e., the hole left following ionization [9-12]. The angle-resolved yields show that ionization is suppressed for cases where the laser polarization lies along the nodal plane of the molecule as a result of destructive interference of the outgoing electron wave [13]. This observation has been used to follow dissociation dynamics in a diatomic molecule [14]. In addition to the influence of orbital symmetry (i.e., the location of nodal planes), the extent to which the wave function samples the saddle point of the potential may also play a role in the ionization yield [15].

In the case of polyatomic molecules, the size of the molecule has been shown to play an important role in the

Published by the American Physical Society under the terms of the Creative Commons Attribution 3.0 License. Further distribution of this work must maintain attribution to the author(s) and the published article's title, journal citation, and DOI. ionization yield [16], although it is not yet clear to what extent the ionization yield as a function of the angle yields information about the shape of molecular orbitals. An important issue is electron correlation, which may lead to a breakdown of Koopmans' correlations between neutral and ionic electronic configurations [17]. These have proven to be very useful in time-resolved measurements, which make use of single-photon or weak-field ionization [18]. Calculations that take electron correlation into account and go beyond the strong-field approximation are now beginning to address this $[19,20]$.

In this work, we study the angle- and time-resolved ionization of excited uracil molecules into different final states of the molecular cation in an effort to isolate the contributions of individual molecular orbitals, and relate the angle-resolved ionization yields to the shape of the relevant orbitals. A deep ultraviolet (UV) pump pulse $\left(\lambda_{0}=\right.$ $262 \mathrm{~nm}$ ) prepares the molecules in the first bright excited state of the molecule $\left(\mathrm{S}_{2}\right)$, and is followed by a strong-field pulse in the near infrared (IR) $\left(\lambda_{0}=780 \mathrm{~nm}\right)$. The pump pulse leaves the sample of excited molecules partially aligned through selective excitation as molecules whose $\mathrm{S}_{0} \rightarrow \mathrm{S}_{2}$ transition dipole moments (TDM) are aligned with the pump-pulse polarization are preferentially excited. The TDM for the $\mathrm{S}_{0} \rightarrow \mathrm{S}_{2}$ transition in uracil is shown in Fig. 1. The probability of exciting any molecule in the randomly oriented sample is proportional to the square of the cosine of the angle between the pump-pulse polarization vector and the $S_{0} \rightarrow S_{2}$ TDM. The full time-of-flight mass spectrum (TOFMS) for fragment ions produced by the probe pulse is measured as a function of the pump-probe delay and angle. We use $a b$ initio electronic structure calculations to obtain the energies and electronic configurations of the neutral and ionic states, as well as energetic requirements for fragmentation from accessible ionic states. Our calculations allow us to associate fragments in the TOFMS with the final states of the cation, and to compare the energies and electronic configurations of the neutral and ionic states. 


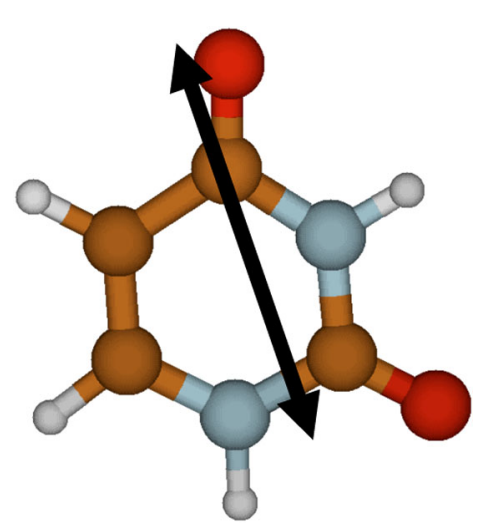

FIG. 1. Geometry of ground-state uracil and transition dipole moment for the $S_{0} \rightarrow S_{2}$ electronic transition.

\section{EXPERIMENTAL APPARATUS}

Our amplified Ti:sapphire laser system produces $30 \mathrm{fs}$ pulses in the near IR $\left(\lambda_{0}=780 \mathrm{~nm}\right)$ at a repetition rate of $1 \mathrm{kHz}$ and with an energy of $1 \mathrm{~mJ}$. The laser pulses are directed into a bichromatic Mach-Zender interferometer in order to generate the pump and the probe pulses with a variable time delay. Approximately $20 \%$ of the laser output is directed into the probe arm, which contains a delay stage and a variable ND filter used to vary the delay and energy of the IR probe pulses. The other arm of the interferometer contains a series of nonlinear crystals used to generate the pump pulses at about $262 \mathrm{~nm}$. The pump pulses traverse an AOM-based pulse shaper [21] and half-wave plate, which allow us to control the power, polarization, and shape of the pump pulses. The pump pulses from our pulse shaper have a pulse duration below $50 \mathrm{fs}$ and a pulse energy as high as $2.5 \mu \mathrm{J}$. The peak intensities of the pulses were about $0.3 \mathrm{TW} / \mathrm{cm}^{2}$ for the UV (low enough to avoid saturating the $\mathrm{S}_{0}-\mathrm{S}_{2}$ transition or producing any ion signal) and about $10 \mathrm{TW} / \mathrm{cm}^{2}$ for the IR. The experimental setup is described in more detail in [22].

Powdered uracil is sublimated in a stainless steel oven at $140^{\circ}-150^{\circ} \mathrm{C}$, and introduced into the vacuum chamber as an effusive molecular beam through a heated, continuously open $0.5 \mathrm{~mm}$ nozzle. The laser beams are focused into the molecular beam inside a time-of-flight mass spectrometer with dual slope extraction and a mass resolution $\Delta \mathrm{m} / \mathrm{m}$ greater than 200 .

\section{CALCULATIONS}

Doublet ionic states for uracil were calculated using the multireference perturbation theory (MRPT2) method $[23,24]$, perturbing one state at a time. Molecular orbitals from a state-averaged, complete-active-space selfconsistent-field calculation with a complete active space $(13,10)$ which includes all eight $\pi$ orbitals and two lonepair orbitals from the two oxygen atoms were used. Eight ionic states were averaged. This active space is sufficient to describe all the low-lying ionic states since it includes all the valence, high-level $\pi$ and lone-pair orbitals from which the electron is most likely to be removed. The 6-311+G(d,p) basis set was used. More details about the cationic states can be obtained in the original publication [25].

The singlet neutral excited states and the corresponding transition dipole moment were calculated using a multireference configuration interaction (MRCI) wave function. The orbitals used in the MRCI were taken from a stateaveraged complete active space self-consistent field calculation with an active space of 12 electrons in 9 orbitals, denoted as (12,9), including all eight $\pi$ and one lone-pair orbital from the oxygen atom. It has been found that the second lone pair is not important to describe the first two singlet excited states [26]. The MRCI expansion included single excitations out of this active space. Including all double excitations is prohibitive for such a large system. Details have been published previously [26]. The transition dipole moment was calculated at the MRCI level since this property was not implemented at the MRPT2 level. However, for the calculation of the ionic states, MRPT2 was preferred over MRCI, as the former includes a more dynamical correlation in this case and is thus expected to perform better than the latter. This is because MRCI here only includes single excitations. The dynamical correlation is usually more balanced between the open-shell ionic states, and MRPT2 is quite appropriate to describe these states.

The calculations reported here were conducted using the computational packages COLUMBUS [27] and GAMESS [28]. Visualization was rendered with MACMOLPLT [29] and MOLDEN [30].

\section{RESULTS AND DISCUSSION}

Figure 2 shows the time-of-flight mass spectrum of uracil at positive and negative pump-probe delays. In the case of negative delays (probe-pump), the intense IR pulse probes the ground state $\left(\mathrm{S}_{0}\right)$ of the molecule and is accountable for all of the ionization signals observed. For ionization of the molecular ground state at probe-pulse intensities well below saturation, the ionization is dominated by removal of an electron from the least bound, or highest occupied molecular orbitals (i.e. HOMO, HOMO-1, ...) [31], and projects the molecule onto the lowest states of the cation $\left(\mathrm{D}_{0}, \mathrm{D}_{1}, \ldots\right)$. According to our calculations, $\mathrm{D}_{0}$ and $\mathrm{D}_{1}$ are not dissociative when produced at the Franck-Condon point, and lead only to the parent ion. However, the next several lowest lying states predominantly produce the fragment at $69 \mathrm{amu}\left(\mathrm{C}_{3} \mathrm{NOH}_{3}^{+}\right)$upon dissociation. Based on energy barriers to dissociation, fragment 69 is the easiest to produce, requiring about $2 \mathrm{eV}$ above the minimum of the $\mathrm{D}_{0}$ [32]. Our measurements for negative pump-probe delays are consistent with this, showing the parent ion and 69 amu dominating the TOFMS. For IR intensities even lower than that used for the 


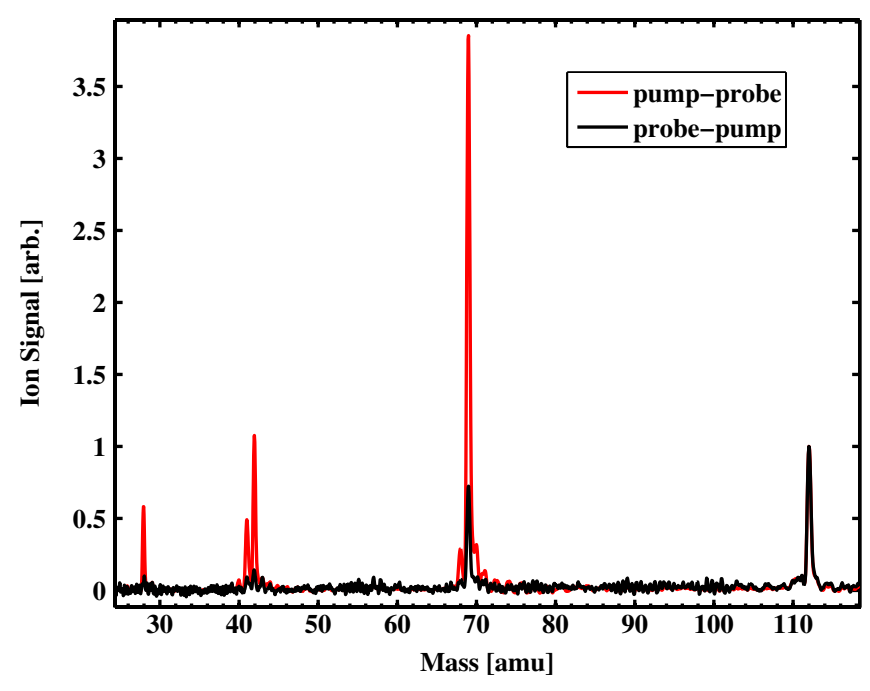

FIG. 2. Time-of-flight mass spectra of uracil for negative and positive time delays $(+/-200 \mathrm{fs})$. Both spectra are normalized to the parent-ion yield.

measurements shown in Fig. 2 the TOFMS shows no ions other than the parent ion, which is consistent with ionization to the two lowest ionic states, $\mathrm{D}_{0}$ and $\mathrm{D}_{1}$. As the UV pulse follows the ionization pulse at negative delays, these data show no angular dependence.

For positive time delays, the total ionization yield increases significantly, as the ionization potential of the molecule in $S_{2}$ is much lower than in $S_{0}$. In addition, the fragmentation pattern shifts towards the production of smaller fragments in the TOFMS-most notably 28 $\left(\mathrm{H}_{2} \mathrm{CN}^{+}\right), 41\left(\mathrm{H}_{3} \mathrm{C}_{2} \mathrm{~N}^{+}\right)$, and $42\left(\mathrm{H}_{2} \mathrm{C}_{2} \mathrm{O}^{+}\right)$amu. The association of structures with the masses measured in our TOFMS is discussed in [32]. The shift in the mass spectrum is evident from Fig. 2, but this figure does not reflect the increase in ion yields since both spectra shown in it are normalized to the parent ion. The top panel of Fig. 3 shows how the yields of several fragments increase for positive delays relative to negative ones.

The time- and angle-dependent yields of 28, 41, and 42 are shown in Fig. 3. The angle and time dependence of the yields for 28, 41, and 42 are very similar, although they are distinct from the yields for other fragments such as the parent and 69. These three fragments are thus produced from the same ionic state or states. In order to determine which cationic state(s) of the molecule can lead to the production of these fragments, we calculated the dissociation energies and barriers to dissociation for these fragments on the ground ionic state of the molecule. Given a high density of ionic states, a molecule ionized to a higher ionic state will tend to undergo a rapid radiationless decay to $\mathrm{D}_{0}$, and dissociation will proceed from the vibrationally hot $\mathrm{D}_{0}$ potential-energy surface. This fast radiationless decay is similar to that occurring in neutral molecules upon excitation. Even if highly excited states are accessed, fluorescence most often originates from the first excited
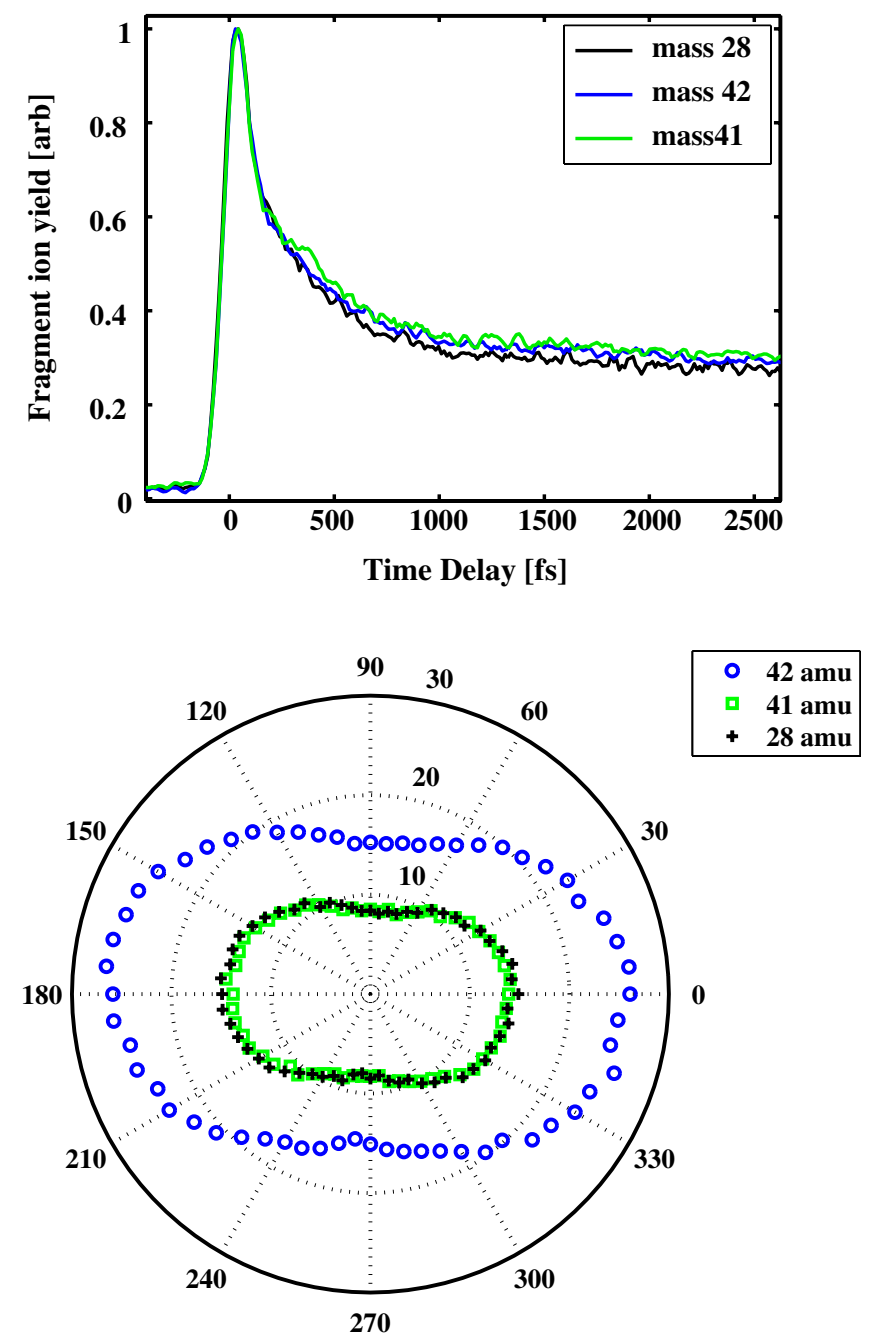

FIG. 3. Top panel: Pump-probe signals from 42, 41, and 28 amu fragments. The signal for each fragment is normalized. Bottom panel: Pump-probe ion yield vs pump polarization at $200 \mathrm{fs}$ pump-probe delay. The azimuth angle corresponds to the relative polarization between the pump and the probe beams, while the radial distance is the ion count in arbitrary units.

state after relaxation to it. This is a common occurrence in photochemistry, called Kasha's rule, first proposed by Kasha in 1950 [33]. The electronic energies of the excited ionic states indicate how much energy will be stored in the cation after ionization to these states. Radiationless decay will convert electronic energy to vibrational energy on $\mathrm{D}_{0}$, which can then be used to dissociate the cation.

While radiationless decay may, in principle, compete with rapid fragmentation in an excited state of the ion, many of the fragmentation energies we calculate are comparable to the difference in energy between the excited and ground states, implying that dissociation on the excited states is not energetically favorable. Furthermore, parallel experiments that we have carried out on other molecules with a velocity-map-imaging apparatus show that ionization to excited states of the cation is predominantly 
followed by dissociation on the ground state of the cation, where fragments are ejected with the maximum possible kinetic energy [34]. Finally, we note that if the molecule does not relax all the way to the ground state prior to dissociation, we expect the barrier to dissociation to be comparable to the ground-state-dissociation barrier in order to avoid curve crossings that would facilitate relaxation to the ground state prior to dissociation.

The calculated energies of the first six excited ionic states are $0.44,0.98,1.40,3.01,4.01$, and $4.18 \mathrm{eV}$ above $\mathrm{D}_{0}$ at vertical ionization $\left(\mathrm{S}_{0}\right.$ minimum $)$ for $\mathrm{D}_{1}, \mathrm{D}_{2}, \mathrm{D}_{3}, \mathrm{D}_{4}$, $\mathrm{D}_{5}$, and $\mathrm{D}_{6}$, respectively, $[25,32]$. The calculated barriers and measured appearance energies [35] for creating fragments 28,41 , and 42 are above $3 \mathrm{eV}$ [32], indicating that these fragments cannot be created from states below $\mathrm{D}_{5}$. Fragmentation in $D_{5}$ and $D_{6}$ is energetically allowed and expected since these states lie over $4 \mathrm{eV}$ above $\mathrm{D}_{0}$ and this energy can be used for dissociation over the barriers. As the variation in yields of these fragments with the time delay and angle is not sensitive to the IR laser intensity around the value for the measurements in Fig. 3, and we worked at the lowest IR intensity where the fragment yields had a sufficient signal-to-noise ratio, we argue that the fragment ion yields are dominated by the lowest state which can dissociate to form them. In this case, we consider both $\mathrm{D}_{5}$ and $D_{6}$ as the possible source of these fragments since they are nearly degenerate and have electron configurations that correspond to removing an electron from a single orbital of $\mathrm{S}_{2}$.

Projecting a final ( $\mathrm{N}-1$ electron) ionic state onto the initial ( $\mathrm{N}$ electron) state of the neutral molecule yields a Dyson orbital of the $n$-th ionization channel: $\psi_{\text {Dyson }}=$ $\left\langle\psi_{0}^{\mathrm{N}}\left(r_{1}, r_{1}, \ldots, r_{\mathrm{N}}\right) \mid \psi_{n}^{\mathrm{N}-1}\left(r_{2}, \ldots, r_{\mathrm{N}}\right)\right\rangle$, where $\psi_{0}^{\mathrm{N}}$ and $\psi_{n}^{\mathrm{N}-1}$ are the wave functions of the neutral and the ionic states. Figure 4 shows the configurations for the neutral excited states as well as the ionic states. $\mathrm{S}_{2}$ can be described as a HOMO- $\rightarrow$ LUMO excitation. In the case of uracil, the Dyson orbitals for ionization of $S_{2}$ to the ionic states of interest have high degrees of overlap with individual molecular orbitals of the neutral molecule, i.e., removal of an electron from a single orbital leads to a single predominant ionic state. Projecting $\mathrm{S}_{2}$ onto $\mathrm{D}_{5}$ or $\mathrm{D}_{6}$ corresponds to removing an electron from the HOMO-2 or HOMO orbitals, respectively. In other words, removal of an electron from HOMO-2 or HOMO in $\mathrm{S}_{2}$ leads to $\mathrm{D}_{5}$ or $\mathrm{D}_{6}$, respectively. In contrast, removal of the LUMO electron from $S_{2}$ leads to a state with a hole in HOMO-1, which correlates well with the ground state of the ion $\mathrm{D}_{0}$.

While projecting $S_{2}$ onto specific final ionic states allows us to identify orbitals from which an electron is removed, the magnitude of the Dyson orbitals are only formally related to the ionization yield to a specific state in the limit of single-photon ionization. Strong-field ionization from $S_{2}$ to $D_{1}$ is, in principle, possible, even though the magnitude of the Dyson orbital corresponding to this

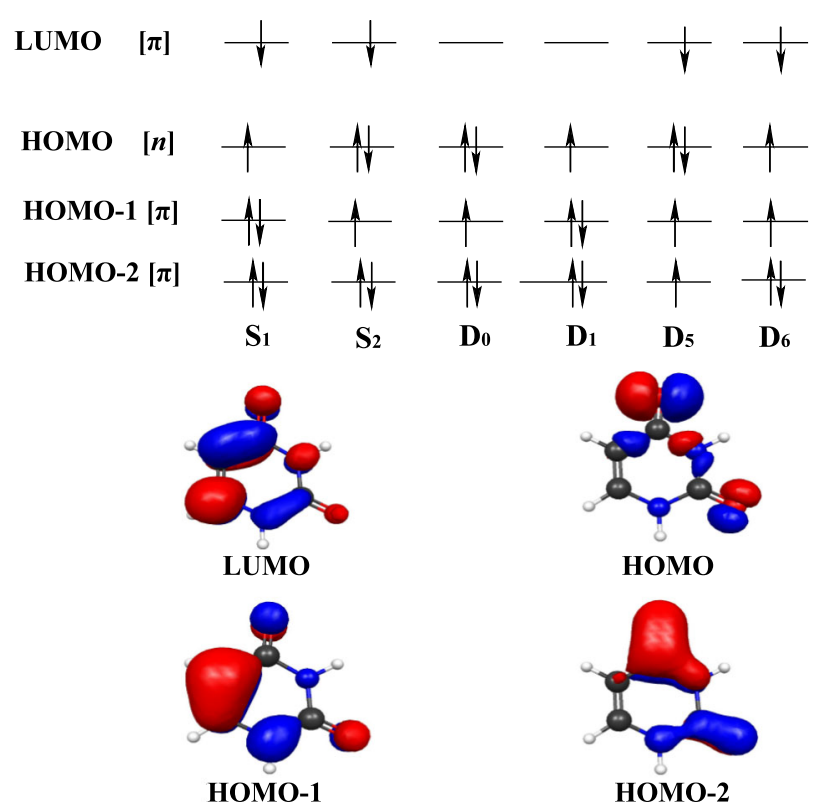

FIG. 4. Dominant electronic configuration and characters for relevant states of the neutral molecule and the ion. The participating orbitals are shown as well. The orbitals are shown for the molecule in the same orientation as shown in Fig. 1.

ionization channel is small (i.e., $\mathrm{S}_{2}$ and $\mathrm{D}_{1}$ are not well correlated, as shown in Fig. 4). However, the largest contribution to the parent signal will come from $\mathrm{D}_{0}$, and we expect that any angular dependence of the ionization yields will be given by the LUMO orbital given the low probe intensities we used and the lack of intensity dependence for the angle-resolved parent-ion yields.

Having established a correspondence between ionic fragments and final cationic states, we now look at the measured angle-dependent yields for the parent and fragment ions to see if they reflect the shape of the orbitals that are determined through projecting $\mathrm{S}_{2}$ onto $\mathrm{D}_{0}$ and $\mathrm{D}_{5} / \mathrm{D}_{6}$. Figure 5 shows angle-resolved pump-probe ion yields for the parent ion and 42 at several positive pumpprobe delays. Angles of $0^{\circ}$ and $180^{\circ}$ correspond to parallel relative polarization between the pump and the probe beams, while $90^{\circ}$ and $270^{\circ}$ correspond to perpendicular relative polarization between the two beams. Experiments carried out with the probe polarized in the horizontal and the vertical plane yielded the same dependence on the relative polarization between the pump and probe. The data is an average of several $(\sim 20)$ polarization scans taken in succession. The ion yield at each angle in the $0^{\circ}-360^{\circ}$ range was measured independently.

The angular dependence of the ionization is expected to be influenced by the shape of the orbital from which an electron is removed to yield a product observed in the mass spectrum. In this work we compare the direct ionization through the removal of a high-lying LUMO electron, which produces the parent ion, and the ionization through the removal of an electron from a more deeply 

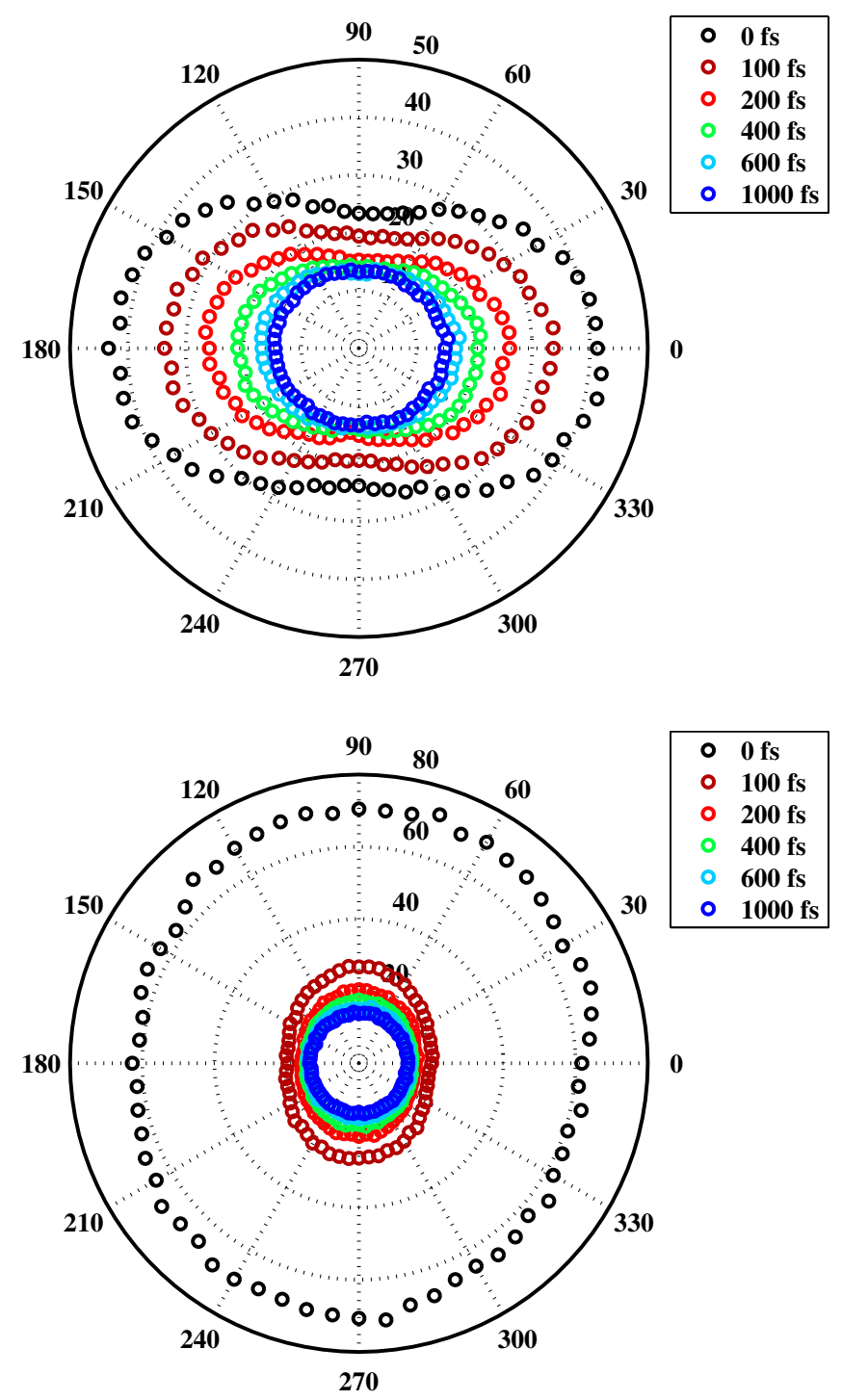

FIG. 5. Pump-probe ion yield vs pump polarization and pumpprobe delay. Top panel: For 42 amu. Bottom panel: For the parent ion. The azimuth angle corresponds to the relative polarization between the pump and the probe beams, while the radial distance is proportional to the ion yield in arbitrary units. Both the parent ion and 42 amu show a decay in the yield with increasing pump-probe delay.

bound orbital, which leads to the production of lighter fragments.

The LUMO orbital is $\pi$-like, having contributions from the electron density on both sides of the molecular plane (there is a nodal plane in the plane of the molecule, in which the TDM lies; see Figs. 1 and 4). Based on measurements of ionization by removal of the most weakly bound electron in diatomic and linear triatomic molecules [9-11], we expect suppression of ionization in the nodal plane of the LUMO orbital. As is evident from Fig. 5, the creation of the parent ion from the LUMO orbital is indeed suppressed when the polarizations of the pump and probe pulses are parallel. Given that the TDM lies in the $\pi$ nodal plane, our measurements show that ionization of the LUMO is suppressed along the plane of the molecule $\left(0^{\circ}\right.$ and $\left.180^{\circ}\right)$ relative to the direction perpendicular to the plane of the molecule $\left(90^{\circ}\right.$ and $\left.270^{\circ}\right)$.

The case of fragments 28,41 , and 42 is more complicated, as suppression along the principal nodal plane of either orbital is not obvious. According to our previous discussion, these fragments are expected when ionization leads to the higher ionic states $\mathrm{D}_{5}$ and $\mathrm{D}_{6}$. In the case that these fragments arise from removal of a HOMO electron, associated with the $\mathrm{D}_{6}$ ionic state, the nodal plane of the main lobe of this orbital contains the $\mathrm{S}_{0}-\mathrm{S}_{2}$ TDM. In this case one would expect suppression of the ionization parallel to the TDM. In the case of ionization through the removal of an electron from HOMO-2, associated with the $\mathrm{D}_{5}$ state, the principal nodal plane is clearly parallel to the TDM. There is a second nodal plane for HOMO-2, which is perpendicular to the TDM. Suppression in this second nodal plane could contribute to the favoring of ionization parallel to the TDM, although it is not clear why the suppression in the second nodal plane should be stronger than in the primary one parallel to the TDM. Thus, it seems that the observed ionization from HOMO or HOMO-2 does not lend itself to a simple explanation in terms of the symmetry of the orbitals.

The limited contrast between the ionization yields for perpendicular and parallel polarizations, and the lack of detailed structure are a result of several limitations in our measurements. These include the fact that while the molecules are preferentially excited with their TDMs along the pump polarization vector, this only constrains the molecule along one angle, and does not result in three-dimensional alignment [36]. Furthermore, the measurement is proportional to a convolution of the actual ionization probability as functions of the angle with the distribution of molecules vs the angle [9]. Finally, there is rotational dephasing leading to random molecular alignment on a timescale of about $1 \mathrm{ps}$ for our molecular ensemble at $\sim 130^{\circ} \mathrm{C}$.

A "net" ionization of a particular orbital can be interpreted in several ways. For example, the ionization via the loss of an electron from a HOMO orbital can be accomplished through the direct removal of a HOMO electron, or indirectly, through the removal of a lower-lying electron followed by a relaxation of a HOMO electron to take its place. Another way to describe this in a multielectron picture is in terms of relaxation from a higher-lying ionic state to lower ones. This relaxation, or coupling between ionic states can be due to field-free terms of the molecular Hamiltonian (e.g., via a conical intersection between ionic states), or it may be driven by the laser field during ionization, particularly if the spacing between states is close to the laser frequency, i.e., near resonant interaction [37]. We argue that fragment ions, which can be produced by the removal of an electron from a given molecular orbital with multiple contributions, should not necessarily have an 
angular distribution which reflects the shape or symmetry of the orbital in which a hole is created at the end of the interaction, since there can be multiple contributions to the production of a hole in a given molecular orbital. However, the parent-ion should still have the angular distribution dictated by the LUMO orbital, since the removal of a LUMO electron from the $S_{2}$ configuration is not only the most probable process, but also one that can occur without any configuration changes. Ionization of a LUMO electron from $S_{2}$ leading to $D_{0}$ is not expected to have contributions from several configurations which can complicate the angular distribution, because the final cationic state is $\mathrm{D}_{0}$, from which no relaxation in the ion is possible as it is the lowest energy state. Furthermore, any laser-driven coupling between ionic states would result in the molecule being left in a dissociative excited ionic state, and would therefore not lead to parent-ion production which is associated with the LUMO orbital.

Our measurements suggest that the ionization of a molecule via the removal of the most weakly bound electron to produce the parent ion should be dictated by the shape of the orbital from which that electron is removed, but the removal of electrons from more deeply bound orbitals can be complicated by configuration changes during ionization, which can involve multiple orbitals in the ionization process. Configuration changes in the ionization associated with removal of the most weakly bound electron are not energetically allowed, whereas such changes are energetically favorable in the case of ionizing by removal of more deeply bound electrons. It is therefore natural to expect that electron correlation plays a more significant role in the removal of a HOMO or deeper bound electron as compared with a LUMO or above. This is consistent with our measurement of the parent-ion angular distribution following the symmetry of the LUMO, whereas fragments resulting from the removal of more deeply bound electrons do not simply follow the symmetry of the associated orbital.

Our discussion has focused on measurements of the angular distributions for small positive time delays, when the wave packet launched on $S_{2}$ by the pump pulse is still near the Franck-Condon region. With increasing time delays, the wave packet can relax down to $S_{1}$ and $S_{0}$, and a detailed analysis of the time-dependent angularly resolved ion yields may hold important information about this relaxation. A detailed analysis of the time-dependent angular distributions for uracil and other molecules will be the subject of a future manuscript.

As a further test of our conclusion that the angular dependence for the ionization by removal of the most weakly bound electron from an excited-state molecule should follow the symmetry of the molecular orbital, we performed angle- and time-resolved measurements of the ionization of excited $\mathrm{CH}_{2} \mathrm{BrI}$ with the same apparatus. For this molecule, at short delays one expects a maximum in the angle resolved parent-ion yield at zero degrees, given the $\mathrm{S}_{0}-\mathrm{S}_{1}$ TDM and molecular orbitals [38]. Our measurements agree with this expectation. These measurements and their detailed analysis are the subject of a future publication.

\section{CONCLUSION}

In conclusion, we have presented time and angleresolved measurements of strong-field ionization of excited-state uracil molecules. By combining our measurements with $a b$ initio electronic structure calculations, we are able to establish a link between fragments in the TOFMS and final states of the molecular ion. By projecting the initial excited state onto the final states of the molecular cation, we can isolate molecular orbitals from which an electron was removed to produce different fragment ions. We find that the angular distribution for ionization via a LUMO electron follows the symmetry of the molecular orbital, whereas ionization involving deeper bound orbitals is more complicated, and a detailed description of the ionization process likely requires taking electron correlation into greater account.

\section{ACKNOWLEDGMENTS}

We gratefully acknowledge insightful discussions with Albert Stolow and Michael Spanner as well as support from the Department of Energy under Grants No. DEFG02-08ER15983 and No. DE-PS02-08ER08-01 and the National Science Foundation under Grants No. PHY0854922 and No. CHE-0911474.

[1] J. Itatani, J. Levesque, D. Zeidler, H. Niikura, H. Pépin, J.C. Kieffer, P. B. Corkum, and D. M. Villeneuve, Tomographic Imaging of Molecular Orbitals, Nature (London) 432, 867 (2004).

[2] S. Haessler, J. Caillat, W. Boutu, C. Giovanetti-Teixeira, T. Ruchon, T. Auguste, Z. Diveki, P. Breger, A. Maquet, B. Carré, R Taïeb, and P. Salieres, Attosecond Imaging of Molecular Electronic Wavepackets, Nature Phys. 6, 200 (2010).

[3] E. Schwartz, Measuring Orbitals: Provocation or Reality?, Angew. Chem. 45, 1508 (2006).

[4] E. Goulielmakis, Z.H. Loh, A. Wirth, R. Santra, N. Rohringer, V.S. Yakovlev, S. Zherebtsov, T. Pfeifer, A.M. Azzeer, and M.F. Kling et al., Real-Time Observation of Valence Rlectron Motion, Nature (London) 466, 739 (2010).

[5] M.F. Kling and M.J.J. Vrakking, Attosecond Electron Dynamics, Annu. Rev. Phys. Chem. 59, 463 (2008).

[6] S. Baker, J. S. Robinson, C. A. Haworth, H. Teng, R. A. Smith, C. C. Chirila, M. Lein, J.W. G. Tisch, and J.P. Marangos, Probing Proton Dynamics in Molecules on an Attosecond Time Scale, Science 312, 424 (2006).

[7] H. Stapelfeldt and T. Seideman, Colloquium: Aligning Molecules with Strong Laser Pulses, Rev. Mod. Phys. 75, 543 (2003). 
[8] S. Patchkovskii, Z. Zhao, T. Brabec, and D. M. Villeneuve, High Harmonic Generation and Molecular Orbital Tomography in Multielectron Systems: Beyond the Single Active Electron Approximation, Phys. Rev. Lett. 97, 123003 (2006).

[9] D. Pavičić, K. F. Lee, D. M. Rayner, P. B. Corkum, and D. M. Villeneuve, Direct Measurement of the Angular Dependence of Ionization for $\mathrm{N}_{2}, \mathrm{O}_{2}$, and $\mathrm{CO}_{2}$ in Intense Laser Fields, Phys. Rev. Lett. 98, 243001 (2007).

[10] A.S. Alnaser, S. Voss, X. M. Tong, C. M. Maharjan, P. Ranitović, B. Ulrich, T. Osipov, B. Shan, Z. Chang, and C. L. Cocke, Effects of Molecular Structure on Ion Disintegration Patterns In Ionization of $\mathrm{O}_{2}$ and $\mathrm{N}_{2}$ by Short Laser Pulses, Phys. Rev. Lett. 93, 113003 (2004).

[11] O. Smirnova, S. Patchkovskii, Y. Mairesse, N. Dudovich, and M. Y. Ivanov, Strong-Field Control and Spectroscopy of Attosecond Electron-Hole Dynamics in Molecules, Proc. Natl. Acad. Sci. U.S.A. 106, 16556 (2009).

[12] Z.X. Zhao, X.M. Tong, and C.D. Lin, AlignmentDependent Ionization Probability of Molecules in a Double-Pulse Laser Field, Phys. Rev. A 67, 043404 (2003).

[13] A. Jaron-Becker, A. Becker, and F. H.M. Faisal, Ionization of $\mathrm{N}_{2}, \mathrm{O}_{2}$, and Linear Carbon Clusters in a Strong Laser Pulse, Phys. Rev. A 69, 023410 (2004).

[14] W. Li, A. A. Jaroń-Becker, C. W. Hogle, V. Sharma, X. Zhou, A. Becker, H.C. Kapteyn, and M. M. Murnane, Visualizing Electron Rearrangement in Space and Time During the Transition from a Molecule to Atoms, Proc. Natl. Acad. Sci. U.S.A. 107, 20219 (2010).

[15] B. K. McFarland, J. P. Farrell, P. H. Bucksbaum, and M. Gühr, High Harmonic Generation from Multiple Orbitals in $\mathrm{N}_{2}$, Science 322, 1232 (2008).

[16] M. J. DeWitt and R. J. Levis, Concerning the Ionization of Large Polyatomic Molecules with Intense Ultrafast Lasers, J. Chem. Phys. 110, 11368 (1999).

[17] T. Koopmans, Über die Zuordnung von Wellenfunktionen und Eigenwerten zu den Einzelnen Elektronen Eines Atoms, Physica (Amsterdam) 1, 104 (1934).

[18] V. Blanchet, M.Z. Zgierski, T. Seideman, and A. Stolow, Discerning Vibronic Molecular Dynamics Using TimeResolved Photoelectron Spectroscopy, Nature (London) 401, 52 (1999).

[19] R. Murray, M. Spanner, S. Patchkovskii, and M. Y. Ivanov, Tunnel Ionization of Molecules and Orbital Imaging, Phys. Rev. Lett. 106, 173001 (2011).

[20] M Spanner (private communication).

[21] B. J. Pearson and T. C. Weinacht, Shaped Ultrafast Laser Pulses in the Deep Ultraviolet, Opt. Express 15, 4385 (2007).

[22] M. Kotur, T. C. Weinacht, C. Zhou, K. A. Kistler, and S. Matsika, Distinguishing Between Relaxation Pathways by Combining Dissociative Ionization Pump Probe Spectroscopy and Ab Initio Calculations: A Case Study of Cytosine, J. Chem. Phys. 134, 184309 (2011).

[23] H. Nakano, Quasidegenerate Perturbation Theory with Multicongurational Self-Consistent-field reference functions, J. Chem. Phys. 99, 7983 (1993).

[24] H. Nakano, MCSCF Reference Quasidegenerate Perturbation Theory with EpsteinNesbet Partitioning, Chem. Phys. Lett. 207, 372 (1993).
[25] M. Kotur, T.C. Weinacht, C. Zhou, and S. Matsika, Following Ultrafast Radiationless Relaxation Dynamics With Strong Field Dissociative Ionization: A Comparison Between Adenine, Uracil, and Cytosine, IEEE J. Sel. Top. Quantum Electron. PP99, 1 (2011).

[26] S. Matsika, Radiationless Decay of Excited States of Uracil Through Conical Intersections, J. Phys. Chem. A 108, 7584 (2004).

[27] H. Lischka, R. Shepard, R. M. Pitzer, I. Shavitt, M. Dallos, Th. Müller, P. G. Szalay, M. Seth, G. S. Kedziora, S. Yabushita, and Z. Zhang, High-Level Multireference Methods in the Quantum-Chemistry Program System COLUMBUS: Analytic MR-CISD and MR-AQCC Gradients and MR-AQCC-LRT for Excited States, GUGA Spinorbit CI, and Parallel CI Density, Phys. Chem. Chem. Phys. 3, 664 (2001).

[28] M. W. Schmidt, K. K. Baldridge, J. A. Boatz, S. T. Elbert, M.S. Gordon, J.H. Jensen, S. Koseki, N. Matsunaga, K. A. Nguyen, S. Su, T. L. Windus, M. Dupuis, and J. A. Montgomery, Computation of Conical Intersections by Using Perturbation Techniques, J. Comput. Chem. 14, 1347 (1993).

[29] B. M. Bode and M.S. Gordon, MACMOLPLT: A Graphical User Interface for GAMESS, J. Mol. Graphics Mod. 16, 133 (1998).

[30] G. Schaftenaar and J.H. Noordik, MOLDEN: A Pre- and Post-Processing Program for Molecular and Electronic Structures, J. Comput.-Aided Mol. Des. 14, 123 (2000).

[31] I. V. Litvinyuk, K. F. Lee, P. W. Dooley, D. M. Rayner, D. M. Villeneuve, and P.B. Corkum, AlignmentDependent Strong Field Ionization of Molecules, Phys. Rev. Lett. 90, 233003 (2003).

[32] C. Zhou, S Matsika, M. Kotur, and T.C. Weinacht, Fragmentation Pathways in Uracil Radical Cations, J. Phys. Chem. (to be published).

[33] M. Kasha, Characterization of Electronic Transitions in Complex Molecules, Discuss. Faraday Soc. 9, 14 (1950).

[34] D. Geissler, T. Rozgonyi, P. Marquetand, J. GonzalezVasquez, L. Gonzalez, and T. C. Weinacht, Pulse Shape Dependent Strong Field Ionization Viewed with Velocity Map Imaging, Phys. Rev. A 84, 053422 (2011).

[35] H. W. Jochims, M. Schwell, H. Baumgartel, and S. Leach, Photoion Mass Spectrometry of Adenine, Thymine, and Uracil in the 6-22 eV Photon Energy Range, Chem. Phys. 314, 263 (2005).

[36] J. G. Underwood, B. J. Sussman, and A. Stolow, FieldFree Three Dimensional Molecular Axis Alignment, Phys. Rev. Lett. 94, 143002 (2005).

[37] H. Harada, S. Shimizu, T. Yatsuhashi, S. Sakabe, Y. Izawa, and N. Nakashima, A Key Factor in Parent and Fragment Ion Formation on Irradiation with an Intense Femtosecond Laser Pulse, Chem. Phys. Lett. 342, 563 (2001).

[38] J. Gonzalez-Vázquez, L. González, S. R. Nichols, T. C. Weinacht, and T. Rozgonyi, Exploring Wavepacket Dynamics Behind Strong-Field Momentum-Dependent Photodissociation in $\mathrm{CH}_{2} \mathrm{BrI}$, Phys. Chem. Chem. Phys. 12, 14203 (2010). 